\title{
Prototype abstraction and the rejection of extraneous patterns
}

\author{
DONALD HOMA \\ Arizona State University, Tempe, Arizona 85281 \\ and \\ B HIBBS \\ New College, Sarasota, Florida 33578
}

\begin{abstract}
The abstraction of prototypical information from ill-defined categories was investigated for categories defined by three and six instances. The major question of interest was whether manipulation of category size would facilitate prototype abstraction when only two categories were learned and a default strategy of classification could not be used. To maximize learning difficulty, categories were defined by high-level (7.7-bit) distortions, and transfer was to new patterns at a high or extreme (8.3-bit) level of distortion. Nearly half the transfer patterns belonged to neither of the learned categories, and subjects were allowed to classify transfer patterns into a "junk" category. All subjects were tested immediately and after a delay of 1 week. The results indicated that category size maintained its potent influence on generalization performance. Although both categories deteriorated across the delay, the category defined by six instances continued to allow new patterns to be classified more accurately.
\end{abstract}

The learning of ill-defined categories (Neisser, 1967) has usually entailed explanations involving the abstraction of a prototype, where the abstracted prototype is thought to represent the central tendency of average of those stimuli that define the category. In the typical paradigm (e.g., Posner \& Keele, 1970), the subject is initially presented with a series of distorted stimuli (such as dot patterns) that must be classified into a number of categories, where all stimuli belonging to the same category are generated from the same prototype. Following criterion learning, a series of transfer patterns is given, either immediately or after a delay, where the stimuli are one of four types: (1) old patterns, that is, those patterns that the subject classified during original learning; (2) new patterns that are derivable from one of the prototypes but previously unseen by the subject; (3) the prototype of each category; and (4) random patterns unrelated to any of the categories. It has been shown that the prototype is classified with greater accuracy than any other new pattern, and that classification of the prototype is unaffected by forgetting of the old stimuli that supposedly allowed for abstraction to occur in the first place (Posner \& Keele, 1968, 1970).

More recently, it has been demonstrated that prototype abstraction is enhanced by three variables: (1) cat-

Requests for reprints should be sent to Donald Homa, Department of Psychology, Arizona State University, Tempe, Arizona 85281. We would like to thank T. Wissect and A. Souther for their critical comments on an earlier draft of this manuscript. This research was submitted by B Hibbs in partial fulfillment toward a senior thesis at New College, Sarasota, Florida. egory size, that is, the number of different stimuli classified together during original learning (Homa, Cross, Cornell, Goldman, \& Shwartz, 1973); (2) number of contrasting categories (Homa \& Chambliss, 1975); and (3) variance or distortion level of the stimuli (Homa \& Vosburgh, 1976). It has been suggested that the variance of the stimuli classified together determines the breadth or boundary of a category (Homa \& Vosburgh, 1976 ), whereas category size and the number of contrasting categories allow for the isolation of common features in a category and the isolation of distinctive features among categories, respectively (Homa \& Chambliss, 1975).

The present research focused upon an anomalous result obtained in the experiment by Homa and Chambliss (1975). In their experiment, subjects had to classify either three or six stimuli into two, four, or six categories. With the exception of the two-category condition, it was found that category size facilitated the subsequent classification of new patterns. It was suggested that category size may have been ineffective for the two-category condition because the learning of one category could allow for classification by default into the second category. Hypothetically, category size should regain its effectiveness if the subject is required to identify the transfer patterns in both categories, but where a default strategy cannot be used. To assess this, subjects in the present experiment were required to learn two categories containing three and six stimuli, followed by a transfer task that allowed the use of an optional third category. Specifically, the subject was instructed to assign each transfer stimulus to either of the two cate- 
gories learned earlier, or to a third "junk" category if they thought the pattern belonged to neither category. To encourage the use of the junk category, a substantial percentage of the transfer patterns $(43 \%)$ was generated from random prototypes. Each subject was informed that $20 \%-50 \%$ of all transfer patterns were, in fact, extraneous to the two categories learned earlier. A second major manipulation was the use of two levels of distortion; half the new patterns were high-level distortions $(7.7 \mathrm{bit} / \mathrm{dot})$ and half were extreme level distortions $(8.3 \mathrm{bit} / \mathrm{dot})$. It was predicted that transfer would be enhanced for the larger category size, and that a higher percentage of the new patterns from the smaller category would be assigned to the junk category. Subjects were also tested after a delay of 1 week to assess whether the benefits of category size would be maintained across this delay.

\section{METHOD}

\section{Subjects}

The subjects were 16 undergraduates from New College who received $\$ 1.50 / \mathrm{h}$ for their participation. Each subject was required to return in 7 days for the delayed test session.

\section{Materials and Apparatus}

Construction of the stimuli has been described previously (Posner, Goldsmith, \& Welton, 1967). Briefly, each prototype is defined as the random placement of nine dots on a 30 by 30 matrix; distortions from each prototype are determined by movement of each of the dots according to statistical decision rules. In lieu of the discrete probability rule employed by Posner et al. (1967) to move each dot, all dots were moved by a continuous distribution (approximately normal), such that the average distance moved per dot corresponded to the $7.7 \mathrm{bit} / \mathrm{dot}$ and $8.3 \mathrm{bit} / \mathrm{dot}$ distortions described by Posner et al. (1967). The total stimulus pool consisted of 62 stimuli, 18 distortions from each of two prototypes plus 24 random patterns. For a given prototype, the 18 distortions consisted of 127.7 -bit/dot and $68.3 \mathrm{bit} / \mathrm{dot}$ distortions; the 24 random patterns were composed of $87.7-$ bit/dot distortions from each of three other prototypes. All stimuli were photographed on $35-\mathrm{mm}$ Panatomic-X film and mounted in slides. A Kodak 650 Carousel projector was used to present the slides.

\section{Procedure}

The subject was seated in an adjoining room and informed that a series of dot patterns would be shown, and that the task was to determine which dot patterns were to be grouped together. Patterns were classified into two groups, $\mathrm{A}$ and $\mathrm{B}$, and the subject was informed that the two categories need not contain an equal number of stimuli.

On the study trials, stimuli were shown sequentially from one of four random orders, and learning was self-paced (i.e., the next stimulus was not shown until the subject had verbally indicated a category choice, A or B). Each response was followed, via headphones, by yes/no feedback, whereupon the next stimulus was presented. Each trial (random order) contained nine 7.7-bit/dot distortions, three derived from one prototype and six derived from another prototype. The learning phase was terminated after no errors had occurred on two consecutive trials.

The test phase commenced immediately after criterion learning. The subject was instructed to categorize the forthcoming test stimuli into one of three categories: A, B, or junk. The subject was further informed that: (1) most of the test stimuli would be new; (2) there were approximately the same number of stimuli in the two categories; and (3) between $20 \%$ and $50 \%$ of all stimuli belonged to the junk category. The entire test sequence contained 56 stimuli, 16 from each of the two categories plus 24 from the junk categories. For each category, the 16 stimuli consisted of three old stimuli $(7.7 \mathrm{bit} / \mathrm{dot}), 12$ new stimuli (6 at $7.7 \mathrm{bit} / \mathrm{dot}$ and 6 at $8.3 \mathrm{bit} / \mathrm{dot}$ ), and the prototype. The test stimuli were randomly presented and shown one at a time; following the subject's response, the next stimulus was presented. No feedback was provided at any time during the test phase. All subjects returned 1 week later, and received the same set of 56 test stimuli, but presented in a different random order.

\section{Design}

A within-subjects design was used, with number of stimuli per category (three and six), stimulus type (old, 7.7 new, 8.3 new, prototype, and junk), and time of test (immediate and delay) as the major variables. Across the 16 subjects, each prototype was used equally often to represent the three- and sixinstance categories. Each of the 12 7.7-bit/dot distortions for each category appeared equally often as an old or new stimulus.

\section{RESULTS}

The mean number of trials to criterion was 8.12. Table 1 shows the mean classification performance for each stimulus type (old, 7.7 new, 8.3 new, prototype, junk) as a function of category size $(3,6)$ and time of test (immediate, 1-week delay). As expected, performance on the stimulus types was most accurate for the old stimuli, worst on the new 8.3-bit/dot patterns, and intermediate for the prototype and new 7.7-bit/dot stimuli. The effect of category size was especially marked for the new patterns at both distortion levels, with the six-instance categories exceeding the performance of the three-instance categories on an immediate test by $.282(7.7 \mathrm{bit} / \mathrm{dot})$ and $.302(8.3 \mathrm{bit} / \mathrm{dot})$, and by $.063(7.7 \mathrm{bit} / \mathrm{dot})$ and $.219(8.3 \mathrm{bit} / \mathrm{dot})$ after the delay. These differences were partially modulated by the slightly greater tendency to classify junk patterns into the larger category immediately (.208 vs. .289) but with this bias reversing itself after the delay (.266 vs. .234 ).

After adjustments were made for opportunities for errors (e.g., twice as many new stimuli appeared on the test trials relative to the old), an analysis of variance

Table 1

Mean Classification Performance

\begin{tabular}{|c|c|c|c|c|c|c|c|}
\hline \multirow{3}{*}{$\begin{array}{l}\text { Cate- } \\
\text { gory } \\
\text { Size }\end{array}$} & \multirow[b]{3}{*}{ Stimulus } & \multicolumn{6}{|c|}{ Response } \\
\hline & & \multicolumn{3}{|c|}{ Immediate } & \multicolumn{3}{|c|}{ 1-Week Delay } \\
\hline & & 3 & 6 & Junk & 3 & 6 & Junk \\
\hline 3 & $\begin{array}{l}\text { Old } \\
\text { New (7.7) } \\
\text { New (8.3) } \\
\text { Prototype }\end{array}$ & $\begin{array}{l}.875 \\
.510 \\
.292 \\
.688\end{array}$ & $\begin{array}{l}.042 \\
.250 \\
.281 \\
.250\end{array}$ & $\begin{array}{l}.083 \\
.240 \\
.427 \\
.062\end{array}$ & $\begin{array}{l}.750 \\
.552 \\
.271 \\
.688\end{array}$ & $\begin{array}{l}.104 \\
.167 \\
.250 \\
.125\end{array}$ & $\begin{array}{l}.146 \\
.281 \\
.479 \\
.188\end{array}$ \\
\hline 6 & $\begin{array}{l}\text { Old } \\
\text { New (7.7) } \\
\text { New (8.3) } \\
\text { Prototype }\end{array}$ & $\begin{array}{l}.146 \\
.073 \\
.115 \\
.125\end{array}$ & $\begin{array}{l}.812 \\
.792 \\
.594 \\
.812\end{array}$ & $\begin{array}{l}.042 \\
.135 \\
.302 \\
.062\end{array}$ & $\begin{array}{l}.083 \\
.115 \\
.167 \\
.188\end{array}$ & $\begin{array}{l}.667 \\
.615 \\
.469 \\
.688\end{array}$ & $\begin{array}{l}.250 \\
.271 \\
.365 \\
.125\end{array}$ \\
\hline & Junk & .208 & .289 & .503 & .266 & .234 & .500 \\
\hline
\end{tabular}


revealed that the main effects of stimulus type $[\mathrm{F}(3,45)=23.11, \mathrm{MSe}=2.64, \mathrm{p}<.001]$ and delay $[F(1,15)=4.60, \mathrm{MSe}=3.59, \mathrm{p}<.05]$ were highly significant; the effect of category size approached significance $[\mathrm{F}(1,15)=3.61, \mathrm{MSe}=6.75, \mathrm{p}<.10]$. In addition, the Category Size by Stimulus Type interaction was significant $[\mathrm{F}(3,45)=4.22, \mathrm{MSe}=4.22, \mathrm{p}<.05]$ and was largely due to the facilitation afforded new, but not old, stimuli. Separate tests indicated that, on the immediate test, the 7.7-new and 8.3-new stimuli were both classified significantly better for categories defined by six instances $[t(15)=2.90$ and $t(15)=4.21$, respectively, ps $<.01]$. On the delayed test, however, classification accuracy of the new stimuli was significantly better for the six-instance category only for the 8.3 patterns $[\mathrm{t}(15)=2.76, \mathrm{p}<.05]$.

As indicated in Table 1, all stimuli showed substantial forgetting, with most of the forgetting characterized by an increased tendency to classify patterns into the junk categories. As predicted, a higher percentage of new stimuli were classified as junk if they belonged to the three-instance category (.334 immediately, .380 after a delay; for six-instance categories, these values were .218 immediately and .318 after the delay). In fact, performance on the extreme distortions (new 8.3) fell to chance accuracy on the delayed test for the three-instance category.

Since these analyses failed to consider the slight biases to classify new patterns into the three- and sixinstance categories, performance was converted to $\mathrm{d}^{\prime}$ values using classification of junk stimuli into the three- and six-instance categories as estimates of false alarm rates. Figure 1 shows the average d' values for old, new, and prototype stimuli, both immediately and after a delay, as a function of the three- and six-instance categories. This representation largely mirrors the outcomes for the uncorrected performance, with the exception that differences for the three- and six-instance categories were slightly increased for the delayed test $\left(\mathrm{d}_{3}^{\prime}=.75, \mathrm{~d}_{6}^{\prime}=1.02\right.$ for new $7.7 ; \mathrm{d}_{3}^{\prime}=.01, \mathrm{~d}_{6}^{\prime}=.65$ for new 8.3).

\section{DISCUSSION}

The major prediction was confirmed: When only two categories are learned but a default strategy of classification is eliminated, the facilitative effects of category size on prototype abstraction are obtained. On an immediate test, both the high-level (7.7-bit/dot) and extreme (8.3-bit/dot) distortions were classified with greater accuracy when the category was defined by six exemplars. Both categories suffered substantial forgetting across the 1-week delay, and the benefits of category size tended to dissipate as well, as evidenced by the continued superior classification of only the extreme-level distortions for the larger category. Since previous research has demonstrated that abstracted information can be highly resistant to forgetting for delays as long as 10 weeks (Homa \& Vosburgh, 1976), it seems likely that the degrees of abstraction investigated for the present study represented a limiting case; that is, the learned categories were in a highly labile state. Still, the results of the present study lend additional support for the view that abstraction from ill-

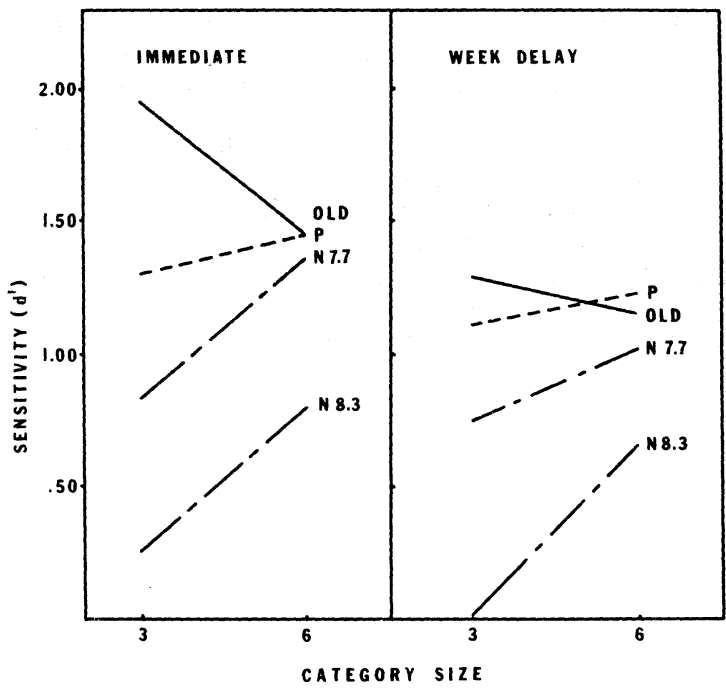

Figure 1. Average $d^{\prime}$ values for old, new, and prototype stimuli, both immediately and after a delay, as a function of categories defined by three and six instances.

defined categories is primarily determined by the amount of exemplar experience.

How might the forgetting of abstracted information be represented? Clearly, the deterioration of a category may involve both an increased tendency to incorporate nonmembers into its domain and an increased tendency to misassign members to other categories. For the six-instance category, fewer junk stimuli were incorporated after the delay, but a substantial increase in the assignment of members to other categories was observed. Interestingly, the majority of these category misassignments took the form of classification into the junk category rather than into the three-instance category. For the weakly abstracted three-instance category, forgetting was characterized by an increased tendency to accept junk patterns, as well as an increased tendency to classify members into the junk category. However, the likelihood of classifying a three-instance member into the six-instance category actually decreased across the delay. A geometric model of abstraction we have been investigating that describes much of this data involved both a concept of a category breadth and category diffusion. Learning variables such as amount and variability of exemplar experience define the breadth of a category, that is, the degree of distortion that a stimulus may undergo and still be accepted for category inclusion. With increasing time delays, however, patterns are "lost" or diffused outward from the original boundary of the category, and the effective breadth of the category is reduced. The better abstracted six-instance category maintains a breadth after the delay (although reduced), since extreme distortions are still acceptable and extraneous patterns can still be rejected. However, the weakly abstracted three-instance category has a poorly defined breadth immediately after learning (e.g., extreme distortions are classified poorly), and with the time delay, the extreme distortions are lost and extraneous patterns cannot be rejected.

\section{REFERENCES}

Homa, D.. \& Chambliss, D. The relative contributions of common and distinctive information on the abstraction from ill-defined categories. Journal of Experimental Psychology: Human Learning and Memory, 1975, 1, 351-359. 
Homa. D., Cross, J., Cornell, D., Goldman, D. \& \& Shwartz. S. Prototype abstraction and classification of new instances as a function of number of instances defining the prototype. Journal Of Experimental Psychology, 1973, 101, 116-122.

Homa, D., \& VosBurgh, R. Category breadth and the abstraction of prototypical information. Journal of Experimental Psychology: Human Learning and Memory, 1976. 2, 322-330.

NEISSER. U. Cognitive psychology. New York: Appleton, 1967.

Posner, M. I., Goldsmith, R., \& Welton, K. E. Perceived distance and the classification of distorted patterns. Journal of Experimental Psychology, 1967, 73, 28-38.
Posner, M. I.. \& Keele, S. W. On the genesis of abstract ideas. Journal of Experimental Psychology, 1968, 77, 353-363.

Posner, M. I.. \& Keele, S. W. Retention of abstract ideas. Journal of Experimental Psychology, 1970, 83. 304-308.

(Received for publication August 1, 1977.) 